\title{
Interobserver and intraobserver agreement of ligamentous injuries on conventional MRI after simple elbow dislocation
}

\author{
Marc Schnetzke ${ }^{1}$, Svenja Schüler ${ }^{2}$, Johannes Hoffend ${ }^{3}$, Rainer Simon ${ }^{1}$, Holger Keil ${ }^{1}$, Felix Porschke ${ }^{1}$,
} Stefan Studier-Fischer ${ }^{1}$, Paul-Alfred Grützner ${ }^{1}$ and Thorsten Guehring ${ }^{1 *}$

\begin{abstract}
Background: The primary objective of this study was to assess the interobserver and intraobserver agreement on ligamentous injuries on conventional magnetic resonance imaging (MRI) in acute simple elbow dislocation. The secondary objectives were to determine the interobserver agreement on the assessment of joint congruity, joint effusion, loose bodies and chondral lesions on conventional MRI.

Methods: Conventional MRIs (1.5 Tesla, elbow specific surface coil) of 30 patients (40.7 years; range 14-72) with simple elbow dislocations were evaluated by four blinded examiners. An analysis of the interobserver agreement of all raters and for several subgroups (radiologists, orthopaedics, experienced, non-experienced) was performed. The examiners assessed the integrity (intact, partial tear, complete tear) of the lateral collateral ligament (LCL), medial collateral ligament $(\mathrm{MCL})$, extensor and flexor tendons, as well as the presence of joint congruity, joint effusion, loose bodies and chondral lesions. Agreement strength, correlation and proportion of exact agreement were determined for interobserver agreement, and intraobserver agreement analyses.

Results: Interobserver agreement of all examiners was fair to moderate for collateral ligaments (LCL: 0.441, MCL: 0.275). Exact agreement of all raters was found in 33.3\% for the $L C L$ and in $26.7 \%$ for the MCL. The both experienced examiners showed highest agreement strength for the LCL (0.619) and the radiologists showed highest agreement strength for the MCL (0.627), the proportion of exact agreement was $60.0 \%$ in both categories. A high proportion of exact agreement regarding joint congruity (90\%), joint effusion (100\%), loose bodies (96.7\%) and chondral lesion (80\%) was found among the radiologists. The evaluation of the intraobserver agreement revealed slight to substantial agreement (0.227 to 0.718 ) for the collateral ligaments.

Conclusions: This study shows difficulties in the evaluation of ligaments by conventional MRI technique as demonstrated by a weak inter- and intraobserver agreement. This should be the basis to develop new MRI quality standards with special focus on coronal oblique reconstructions to improve the evaluation of ligament injuries after simple elbow dislocations.
\end{abstract}

Keywords: Elbow dislocation, Simple elbow dislocation, MRI, Interobserver, Intraobserver, Coronal oblique view, Medial collateral ligaments, Lateral collateral ligaments, Lateral ulnar collateral ligament

\footnotetext{
* Correspondence: guehring@uni-heidelberg.de

${ }^{1}$ Clinic for Trauma and Orthopaedic Surgery, BG Trauma Center

Ludwigshafen at Heidelberg University Hospital, Ludwig-Guttmann-Strasse

13, Ludwigshafen on the Rhine 67071, Germany

Full list of author information is available at the end of the article
}

(c) The Author(s). 2017 Open Access This article is distributed under the terms of the Creative Commons Attribution 4.0 International License (http://creativecommons.org/licenses/by/4.0/), which permits unrestricted use, distribution, and reproduction in any medium, provided you give appropriate credit to the original author(s) and the source, provide a link to the Creative Commons license, and indicate if changes were made. The Creative Commons Public Domain Dedication waiver (http://creativecommons.org/publicdomain/zero/1.0/) applies to the data made available in this article, unless otherwise stated. 


\section{Background}

Simple elbow dislocations are characterised by a severe soft tissue injury with a varying degree of instability [1-7]. The majority of simple elbow dislocations are stable after joint reduction and can be treated by exercises and functional use of the arm within 2 weeks of injury $[3,8,9]$. However, elbow dislocations can result in disabling sequelae, including recurrent instability, posttraumatic contractures and arthritis $[10,11]$. Especially in unstable simple elbow dislocations, adequate treatment is still under discussion $[6,12,13]$. The treatment decision is mainly based on clinical and radiological findings [14]. Various imaging modalities can be used to determine the amount of the soft tissue injury and elbow instability, including ultrasound, plain radiographs, stress radiographs and magnetic resonance imaging (MRI) [12, 15-18]. However, a standardised diagnostic algorithm for acute simple elbow dislocations has not been established yet.

In chronic elbow instability the MRI has been proven to be a valuable diagnostic tool to detect full thickness tears of the collateral ligaments [19]. In acute simple elbow dislocation evidence for the reliability of the MRI is lacking. There are several concerns regarding its interpretation in ligamentous elbow injuries. Partial tears of the medial collateral ligaments (MCL) are detected with a low sensitivity $[19,20]$. Biomechanically relevant structures like the lateral ulnar collateral ligament (LUCL) are not visualised reliably on MRI [21-24]. Furthermore, in acute simple elbow dislocation, most patients are not able to fulfil complete extension of the elbow, which makes interpretation much more difficult because the collateral ligaments are not tensioned $[12,25]$. Therefore, the interpretation of an MRI after elbow dislocation might be difficult. The primary hypothesis of this study was that the interobserver and intraobserver agreement on ligamentous injuries on conventional MRI in acute simple elbow dislocation is weak. Secondary it was hypothesized that a high proportion of exact agreement on the assessment of joint congruity, joint effusion, loose bodies and chondral lesions on conventional MRI can be achieved.

\section{Methods}

In this diagnostic study thirty consecutive patients with the diagnosis of a simple elbow dislocation who underwent an MRI within 4 weeks after injury were included between 2010 and 2015. The definition of simple elbow dislocation included patients with ligamentous injuries of the lateral (LCL) and/or medial collateral ligaments (MCL). Avulsion fractures of the coronoid process type I according to Regan \& Morrey were also included in this study. This definition is in agreement with others [9]. Patients with avulsion fractures of the collateral ligaments, associated articular fractures of the radial head, the olecranon or coronoid fractures type II and III according to Regan \& Morrey were excluded. Patients with previous injuries of the injured elbow were also excluded.

\section{Evaluation of MRI}

The MRIs were examined by two blinded radiologists and two blinded orthopaedic surgeons. The evaluators experience and the average number of weekly-assessed MR examinations of the elbow and other major joints were assessed before the study (Table 1). The principal investigator selected the cases and knew the patients' identities. The physicians evaluating the images were blinded to the diagnosis of patients.

The evaluators had access to the complete examinations, with the full sets of images. In addition, a short medical history and the time between injury and MRI were given to the evaluators. Subsequently, all evaluators were requested to fill out a questionnaire with eight items (Table 2).

All MRIs were performed using 1.5 Tesla scanner with dedicated elbow specific surface coils. In each case, coronal, axial and sagittal images were available in nonfat-saturated T1-weighted and proton density-weighted sequences as well as fat-saturated T2/proton densityweighted or short tau inversion recovery (STIR) sequences. Special MRI reconstructions such as coronal oblique images have not been done. The evaluation of the MRIs was done on a medical viewing monitor with an adjustable brightness and contrast control.

\section{Statistical analysis}

For characterising the study population descriptive statistics (mean, range, absolute and relative frequencies) are reported. An analysis of the interobserver agreement of all raters and for several subgroups (radiologists, orthopaedics, experienced, non-experienced) was performed. Both raters with routine evaluation of 10 or more MRIs/week were defined as experienced (radiologist 1 and orthopaedist 1) and the others that evaluate up to $5 \mathrm{MRI}$ per week have been defined as non-experienced examiners (radiologist 2 and orthopaedist 2).

One option for evaluating the interobserver agreement is to estimate the proportion of exact agreement of all observers. This, however, does not take into account that observers will sometimes agree or disagree simply by chance. The kappa statistics are the most commonly

Table 1 Length of experience and frequency of MRI examination evaluation of the elbow and other joints

\begin{tabular}{llll}
\hline Observer & Experience [y] & Elbow MRIs/week & Joint MRIs/week \\
\hline Radiologist 1 & 29 & $10-15$ & 100 \\
Radiologist 2 & 25 & $1-5$ & 10 \\
Orthopaedist 1 & 15 & $15-20$ & 60 \\
Orthopaedist 2 & 5 & $1-5$ & 10 \\
\hline
\end{tabular}


Table 2 Questionnaire for the assessment of the MRI

\begin{tabular}{lll}
\hline Object & Detailed & $\begin{array}{l}\text { Possible answers } \\
\text { (No. of answers) }\end{array}$ \\
\hline Soft tissue & $\begin{array}{l}\text { Lateral collateral ligament } \\
(\mathrm{LCL})\end{array}$ & $\begin{array}{l}\text { Intact-partial tear- } \\
\text { complete tear (3) }\end{array}$ \\
& $\begin{array}{l}\text { Medial collateral ligament } \\
(\mathrm{MCL})\end{array}$ & \\
& Common extensor tendon & \\
& Common flexor & \\
tendon & Yes-no (2) \\
Others & Joint congruity & \\
& Joint effusion & \\
& Loose bodies & \\
& Chondral lesion &
\end{tabular}

used measure of agreement that take the expected agreement into account [26].

Cohen's kappa was introduced to assess the interobserver agreement of two observers for categorical items. Weighted kappa, a generalised version of Cohen's kappa, was designed to recognise that some disagreements between the two raters are more severe than others and thus weights disagreements differently (e.g. linear or squared). Therefore, it is especially useful for data measured on ordinal scales. In case of more than two raters assigning categorical outcomes, a further modification, Fleiss' kappa is used to assess the agreement.

To evaluate the interobserver agreement of all raters or in the subgroups we therefore calculated Fleiss kappa or weighted kappa (squared weights) values respectively. Spearman's rank correlation coefficients were calculated to identify relations between the different ratings in the subgroups. In addition the proportion of exact agreement was estimated. The interobserver agreement for the LCL and MCL for all observers was also analysed for different joint elbow positions in MRI (flexed vs. extended).

The intraobserver agreement assessed by weighted kappa has been evaluated for the Orthopaedists comparing the results of the same examiner's evaluations at 2 different times. Our target was to perform retests 2 months after baseline assessment. According to literature the time interval should be at least 2 weeks to prevent recall bias [27].

Agreement strength was inferred from kappa index values in accordance with the recommendations of Landis and Koch [28]. Briefly, a kappa value $<0$ was interpreted as poor agreement; a value in the range of 0.01 to 0.20 , slight agreement; a value in the range of 0.21 to 0.40 , fair agreement; a value in the range of 0.41 to 0.60 , moderate agreement; a value in the range of 0.61 to 0.80 , substantial agreement; and a value in the range of 0.81 to 1.00 , nearly perfect agreement. Statistical analysis was performed using $\mathrm{R}$ version 3.1.3.

\section{Results}

The sample consisted of 11 women (36.7\%) and 19 men (63.3\%) with an average age of 40.7 years (range 14 to 72). The left side was affected in 17 patients $(56.7 \%)$ and the right side was affected in 13 patients $(43.3 \%)$. The MRIs were performed 6.3 days (0 to 25) after injury. Twelve MRIs have been performed in our institution and 18 MRI have been performed externally. In 16 patients $(53 \%)$ the elbow was in extended position and in 14 patients (47\%) in flexed position during MRI examination.

The interobserver agreement and Spearman's rank correlation coefficients for all subgroups are reported in Table 3. Interobserver agreement of all examiners was fair to moderate for collateral ligaments (LCL: 0.441, MCL: 0.275). Exact agreement of all raters was found in $33.3 \%$ for the LCL and in $26.7 \%$ for the MCL. The both experienced examiners showed highest agreement strength for the LCL (0.619) and the radiologists showed highest agreement strength for the MCL (0.627), the proportion of exact agreement was $60.0 \%$ in both categories.

An extended elbow position during MRI examination did not improve the interobserver agreement regarding LCL (extended: 0.359, flexed: 0.534) and MCL (extended: 0.284 , flexed: 0.188). For binary variables (joint congruity, joint effusion, loose bodies and chondral lesion) interobserver agreement and Spearman's rank correlation coefficients could not be estimated in most cases due to

Table. 3 Results of interobserver ( $\mathrm{K}_{\mathrm{F}}=$ Fleiss Kappa, $\mathrm{K}_{\mathrm{W}}=$ weighted Kappa) and Spearman correlation (corr) ( ${ }^{*} \mathrm{~K}$ could not be estimated due to spare data), bold values indicate substantial agreement

\begin{tabular}{|c|c|c|c|c|c|c|c|c|c|}
\hline \multirow[t]{2}{*}{$N=30$} & \multirow{2}{*}{$\begin{array}{l}\text { All raters }(n=4) \\
\mathrm{K}_{\mathrm{F}}\end{array}$} & \multicolumn{2}{|c|}{ Radiologists $(n=2)$} & \multicolumn{2}{|c|}{ Orthopaedists $(n=2)$} & \multicolumn{2}{|c|}{ Experienced $(n=2)$} & \multicolumn{2}{|c|}{ Non-Experienced $(n=2)$} \\
\hline & & $K_{w}$ & corr & $K_{w}$ & corr & $K_{w}$ & corr & $K_{w}$ & corr \\
\hline \multicolumn{10}{|l|}{ Ligaments } \\
\hline $\mathrm{LCL}$ & 0.441 & 0.570 & 0.625 & 0.590 & 0.636 & 0.619 & 0.652 & 0.467 & 0.554 \\
\hline $\mathrm{MCL}$ & 0.275 & 0.627 & 0.631 & 0.498 & 0.567 & 0.547 & 0.575 & 0.185 & 0.183 \\
\hline Ext. tendon & 0.049 & 0.093 & 0.133 & 0.309 & 0.215 & 0.363 & 0.323 & 0.089 & 0.212 \\
\hline Flex. tendon & 0.143 & * & 0.043 & 0.175 & 0.090 & 0.351 & 0.359 & 0.121 & 0.119 \\
\hline
\end{tabular}


spare data. A high proportion of exact agreement regarding joint congruity (90\%), joint effusion (100\%), loose bodies (96.7\%) and chondral lesion (80\%) was found among the radiologists (Figs. 1 and 2).

The evaluation of the intraobserver agreement revealed slight to substantial agreement (0.227 to 0.718) for the collateral ligaments (Table 4). Patient examples with discrepancies regarding assessment of the LCL and MCL are shown in Figs. 3 and 4.

\section{Discussion}

The most important finding of the current study was that interobserver agreement of all examiners was only fair to moderate for collateral ligaments (LCL: 0.441, MCL: 0.275) on conventional MRI after acute simple elbow dislocation. Subgroup analysis revealed that the experienced raters showed highest agreement strength for the LCL (0.619) and the radiologists showed highest agreement strength for the MCL (0.627). However, the proportion of exact agreement was still only $60.0 \%$ in both categories. Intraobserver agreement also showed only slight to moderate agreement for the collateral ligaments $(0.188$ to 0.586$)$ with the exception of the rating of the MCL of orthopaedist No. 2 (0.718). The primary hypothesis was proven partially as most agreement analyses revealed only slight to moderate agreement on collateral ligament injuries. These results demonstrate that even for experienced radiologists and orthopaedists, the interpretation of an MRI after acute elbow dislocation might be difficult, particularly for detecting ligamentous injuries.

The number of categories and the grading system of injury will affect the results of any agreement analysis. In the current study, the grading system was adopted from a previous MRI study on the agreement of collateral ligament injuries [19]. However, no standardized classification system for the assessment of collateral ligament injuries on MRI exists. The lack of a classification system might be one reason for the low interobserver and intraobserver agreement found in this study.

There are several studies available regarding the diagnostic value of MRI for detecting tears of the ligamentous structures of the elbow. Previous studies assessing MRI of the elbow are limited by small numbers of patients and small numbers of patients who went on to surgery [19, 20, 29-32]. Furthermore, all previous studies regarding the usefulness of MRI in elbow injuries have been done in cadaveric models or chronic elbow instability.

Carrino et al. found only moderate interobserver agreement for detecting LUCL tears in a cadaveric model and concluded, that radial collateral ligament complex tears can be challenging to see on conventional MRI because of the oblique course of the ligament and the relatively small size of the ligament compared with the ulnar collateral ligament [29].

Carrino et al. conducted another cadaveric study with artificial tears of the MCL and found good agreement (0.78) [30]. Timmerman et al. found MRI to be $100 \%$ sensitive for full-thickness tears of the MCL but only $14 \%$ sensitive for partial-thickness tears in patients with chronic medial elbow pain; the specificity was $100 \%$ [19].

In the current study the LCL and the LUCL were not analysed separately, which would have further decreased the inter- and intraobserver agreement. The assessment of the LUCL can be difficult, and even the intact LUCL is rarely seen as a distinct low-signal band [21].

The assessment of extensor and flexor tendon injuries showed slight interobserver agreement of all raters (Ext. tendon: 0.049, Flex. tendon: 0.143). According to these results, distinguishing between injuries of the muscle tendons and the collateral ligaments seems to be even

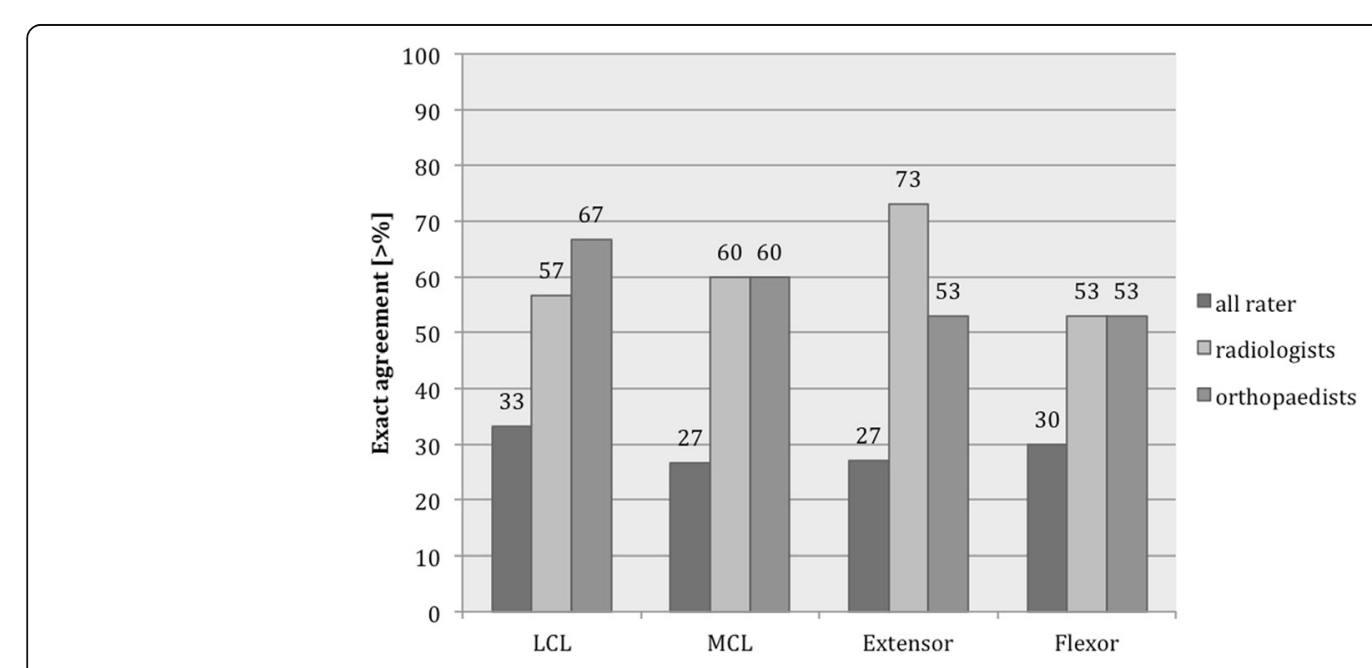

Fig. 1 Proportion of exact agreement [\%] for ligamentous structures 


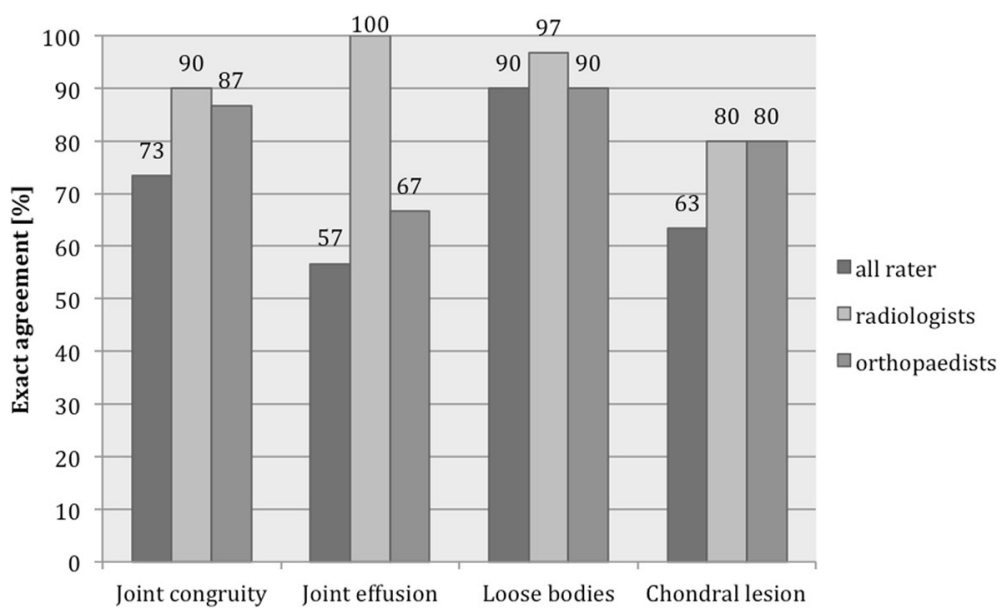

Fig. 2 Proportion of exact agreement [\%] for joint congruity, joint effusion, loose bodies and chondral lesion

more difficult. However, determination of the muscle tendon injuries is an important aspect after simple elbow dislocation, because the muscle tendons are significant stabilisers of the elbow [33].

The discrepancies in the MRI findings regarding the ligamentous structures in our study lead to the question as to whether a conventional MRI is a valuable diagnostic tool in acute simple elbow dislocations. To improve the quality of MRI in acute elbow injury additional planes such as the posterior oblique coronal plane might be useful. In 1997, Cotten et al. investigated a cadaveric study to determine the best plane and position of the elbow for optimal visualization of normal and abnormal collateral ligaments with conventional MRI [34]. The authors concluded that the posterior oblique coronal plane with the elbows extended or the coronal plane aligned with the humeral shaft with the elbows slightly flexed allows accurate assessment of the collateral ligaments. Hill et al. also showed in a cadaveric study, that the medial collateral ligaments of the elbow were best seen on slight posteriorly oblique coronal plane as well [32]. In the current study, posterior oblique coronal views were not used. It should be the focus of further studies investigating the diagnostic accuracy of MRI in acute elbow dislocation using additional coronal oblique

Table 4 Results of intraobserver agreement ( $\mathrm{K}_{\mathrm{w}}=$ weighted Kappa, $\%=$ exact agreement), bold values indicate substantial agreement

\begin{tabular}{llllll}
\hline$N=30$ & \multicolumn{2}{l}{ Orthopaedist 1 } & & \multicolumn{2}{c}{ Orthopaedist 2 } \\
\cline { 2 - 3 } & $K_{w}$ & $\%$ & & $K_{w}$ & $\%$ \\
\hline Soft tissue & & & & \\
LCL & 0.519 & 50 & & $\mathbf{0 . 7 1 8}$ & 73 \\
MCL & 0.440 & 50 & & 0.227 & 46 \\
Ext. tendon & 0.519 & 70 & & 0.485 & 70 \\
Flex. tendon & 0.586 & 70 & & 0.447 & 60 \\
\hline
\end{tabular}

reconstructions. Improving the reliability of the assessment of important primary stabilizes such as the MCL and the LCL on the MRI could help the surgeon to decide on whether to treat the patients by functional rehabilitation alone or by primary surgical stabilization after simple elbow dislocation.

Until now, a diagnostic algorithm for simple elbow dislocation has not been established [12]. Sanchez-Sotelo et al. in 2005 reported that, if the clinical diagnosis is in doubt, a fluoroscopic examination should be performed and occasionally an examination under anesthesia, but other imaging studies such as MRI are usually not

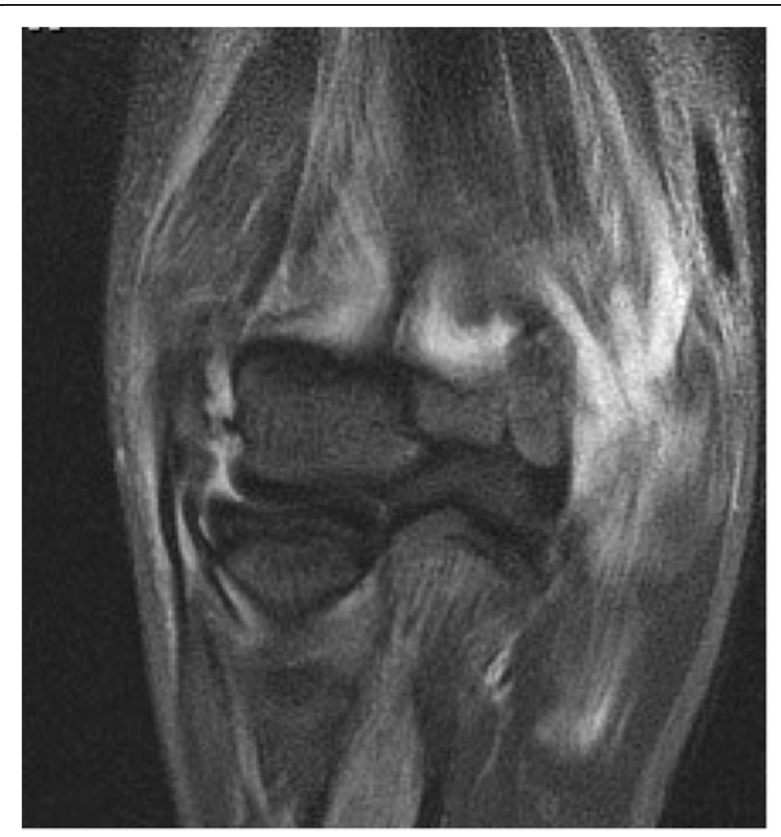

Fig. 3 Coronal view in T2-weighted MRI after simple elbow dislocation: The $L C L$ was rated to be partially torn $(2 x)$ and completely torn $(2 x)$ 


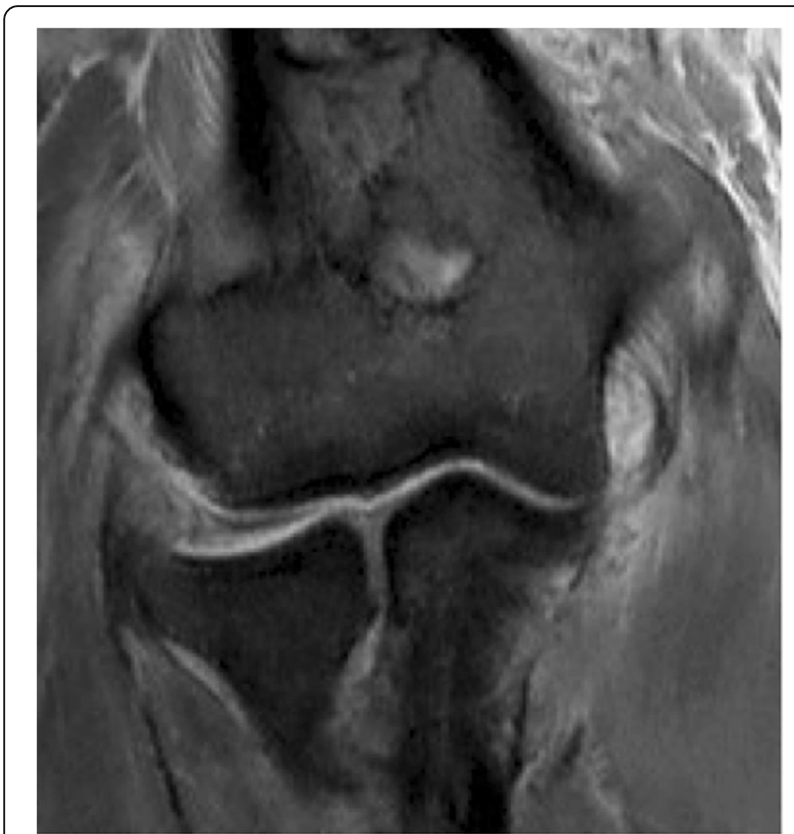

Fig. 4 Coronal view in T2-weighted MRI after simple elbow dislocation: The MCL was rated to be intact $(1 x)$, partially torn $(1 x)$ and completely torn $(2 x)$

needed in simple elbow dislocations [35]. In contrast, Hackl et al. and our study group could show, that it is important to detect possible warning signs of instability on MRI after acute simple elbow dislocation [9, 36]. Our study group could show that the presence of a warning sign of instability (drop sign or joint incongruence) is associated with more complications (Odds ratio 15.9) and higher revision rates (Odds ratio 10.3) after nonoperative treatment of simple elbow dislocations [9]. As hypothesized, a high proportion of exact agreement of all evaluators for joint congruity (73.3\%) and for loose bodies $(90 \%)$ has been found. The radiologist achieved exact agreement for joint congruity in 90\%, for joint effusion in $100 \%$ for loose bodies in $97 \%$ and for chondral lesion in $80 \%$ of cases. This in agreement Hackl et al., who recently reported that tears of the LUCL with resulting posterolateral rotatory instability can be reliably diagnosed on MRI by assessing the joint congruity [36]. These findings support the notion that MRI in combination with clinical finding and stress radiography plays an important role in acute simple elbow dislocation.

This study has several limitations. MRI findings were not compared with intraoperative findings. Therefore, further prospective studies are necessary to evaluate the value of the MRI in simple elbow dislocation and to compare the assessment of the evaluators with intraoperative findings. Another limitation of this study is that intraobserver agreement was only available for the orthopaedic surgeons. In the current study conventional
MRIs without coronal oblique reconstructions were used in all patients, which might have been improved the quality of MRI, and the interobserver and intraobserver agreement of the assessment of the ligamentous structures. Since this study was conducted in one single department, and the assessment was done by only four examiners, the findings may not necessarily be generalizable.

\section{Conclusions}

This study shows difficulties in the evaluation of ligaments by conventional MRI technique as demonstrated by a weak inter- and intraobserver agreement. This should be the basis to develop new MRI quality standards with special focus on coronal oblique reconstructions to improve the evaluation of ligament injuries after simple elbow dislocations.

\section{Abbreviations}

LCL: Lateral collateral ligaments; LUCL: Lateral ulnar collateral ligament; MCL: Medial collateral ligaments; MRI: Magnetic resonance imaging; STIR: Short tau inversion recovery

\section{Acknowledgements}

None.

\section{Funding}

This study is supported by Deutsche Forschungsgemeinschaft and Ruprecht-KarlsUniversität Heidelberg within the funding programme "Open Access Publishing".

\section{Availability of data and materials}

The datasets used and analyzed during the current study are available from the corresponding author on reasonable request.

\section{Authors' contributions}

MS was involved with the design of the study, statistical analysis and manuscript preparation. SS was involved with the data interpretation, statistical analysis and manuscript preparation. JH, RS, HK and TG did the assessment of MRI. TG, FP, SSF and PAG assisted with the data interpretation and manuscript preparation. All authors read and approved the final manuscript.

\section{Authors' information}

TG is senior consultants at the BG Trauma Center Ludwigshafen. PAG is the Medical Director of the BG Trauma Center Ludwigshafen.

\section{Competing interests}

The authors declare that they have no competing interests.

\section{Consent for publication \\ Not applicable.}

Ethics approval and consent to participate

This study was approved by the local ethics committee of the board of Medical Profession of Rhineland-Palatine in Mainz (No. 837.084.14/9323-F). In agreement with the local ethics committee, informed consent was not obtained from the participants because evaluation of data was done retrospectively and anonymously.

\section{Author details}

${ }^{1}$ Clinic for Trauma and Orthopaedic Surgery, BG Trauma Center Ludwigshafen at Heidelberg University Hospital, Ludwig-Guttmann-Strasse 13, Ludwigshafen on the Rhine 67071, Germany. ${ }^{2}$ Institute of Medical Biometry and Informatics, University of Heidelberg, Heidelberg, Germany. ${ }^{3}$ Department for Radiology, Klinikum der Stadt Ludwigshafen gGmbH, Ludwigshafen on the Rhine, Germany. 
Received: 1 November 2016 Accepted: 13 February 2017 Published online: 20 February 2017

\section{References}

1. Boretto JG, Rodriguez Sammartino M, Gallucci G, De Carli P, Ring D. Comparative study of simple and complex open elbow dislocations. Clin Orthop Relat Res. 2014;472(7):2037-43.

2. de Haan J, Schep N, Tuinebreijer W, den Hartog D. Complex and unstable simple elbow dislocations: a review and quantitative analysis of individual patient data. Open Orthop J. 2010;4:80-6.

3. de Haan J, Schep NW, Tuinebreijer WE, Patka P, den Hartog D. Simple elbow dislocations: a systematic review of the literature. Arch Orthop Trauma Surg. 2010;130(2):241-9.

4. Duckworth AD, Ring D, Kulijdian A, McKee MD. Unstable elbow dislocations. J Shoulder Elbow Surg. 2008;17(2):281-6.

5. Englert C, Zellner J, Koller M, Nerlich M, Lenich A. Elbow dislocations: a review ranging from soft tissue injuries to complex elbow fracture dislocations. Adv Orthop. 2013;2013:951397.

6. Hackl M, Beyer F, Wegmann K, Leschinger T, Burkhart KJ, Müller LP. The treatment of simple elbow dislocation in adultsóa systematic review and meta-analysis. Dtsch Arztebl Int. 2015;112(18):311-9.

7. Schnetzke M, Aytac S, Keil H, Deuss M, Studier-Fischer S, Grutzner PA, Guehring T. Unstable simple elbow dislocations: medium-term results after non-surgical and surgical treatment. Knee Surg Sports Traumatol Arthrosc. 2016.

8. Maripuri SN, Debnath UK, Rao P, Mohanty K. Simple elbow dislocation among adults: a comparative study of two different methods of treatment. Injury. 2007;38(11):1254-8.

9. Schnetzke M, Aytac S, Studier-Fischer S, Grutzner PA, Guehring T. Initial joint stability affects the outcome after conservative treatment of simple elbow dislocations: a retrospective study. J Orthop Surg Res. 2015;10:128.

10. Josefsson PO, Johnell O, Gentz CF. Long-term sequelae of simple dislocation of the elbow. J Bone Joint Surg Am. 1984;66(6):927-30

11. Anakwe RE, Middleton SD, Jenkins PJ, McQueen MM, Court-Brown CM. Patient-reported outcomes after simple dislocation of the elbow. J Bone Joint Surg Am. 2011;93(13):1220-6.

12. Hollinger $B$, Dehlinger F, Franke S. Diagnostik und Therapie der ligamentären Ellenbogeninstabilitäten. Obere Extremität. 2014;9(3):147-55.

13. Jeon $I H$, Kim SY, Kim PT. Primary ligament repair for elbow dislocation. Keio J Med. 2008:57(2):99-104

14. Singer AD, Hanna T, Jose J, Datir A. A systematic, multimodality approach to emergency elbow imaging. Clin Imaging. 2016:40(1):13-22.

15. Reichel LM, Milam GS, Sitton SE, Curry MC, Mehlhoff TL. Elbow lateral collateral ligament injuries. J Hand Surg. 2013;38(1):184-201. quiz 201.

16. Rehm J, Zeifang F, Weber MA. Imaging of the elbow joint with focused MRI. Part 1: examination techniques and sequences for bone and ligaments. Radiologe. 2014;54(2):167-80.

17. Teixeira PA, Omoumi P, Trudell DJ, Ward SR, Lecocq S, Blum A, Resnick DL. Ultrasound assessment of the lateral collateral ligamentous complex of the elbow: imaging aspects in cadavers and normal volunteers. Eur Radiol. 2011:21(7):1492-8

18. Konin GP, Nazarian LN, Walz DM. US of the elbow: indications, technique, normal anatomy, and pathologic conditions. Radiographics. 2013:33(4): E125-147

19. Timmerman LA, Schwartz ML, Andrews JR. Preoperative evaluation of the ulnar collateral ligament by magnetic resonance imaging and computed tomography arthrography. Evaluation in 25 baseball players with surgical confirmation. Am J Sports Med. 1994;22(1):26-31. discussion 32.

20. Schwartz ML, Al-Zahrani S, Morwessel RM, Andrews JR. Ulnar collateral ligament injury in the throwing athlete: evaluation with saline-enhanced MR arthrography. Radiology. 1995;197(1):297-9.

21. Hauptfleisch J, English C, Murphy D. Elbow magnetic resonance imaging: imaging anatomy and evaluation. Top Magn Reson Imaging. 2015;24(2):93-107.

22. Sampath SC, Sampath SC, Bredella MA. Magnetic resonance imaging of the elbow: a structured approach. Sports Health. 2013;5(1):34-49.

23. Grafe MW, McAdams TR, Beaulieu CF, Ladd AL. Magnetic resonance imaging in diagnosis of chronic posterolateral rotatory instability of the elbow. Am J Orthop (Belle Mead, NJ). 2003;32(10):501-3. discussion 504.

24. Terada N, Yamada H, Toyama Y. The appearance of the lateral ulnar collateral ligament on magnetic resonance imaging. J Shoulder Elbow Surg. 2004;13(2):214-6.
25. Schreiber JJ, Potter HG, Warren RF, Hotchkiss RN, Daluiski A. Magnetic resonance imaging findings in acute elbow dislocation: insight into mechanism. J Hand Surg. 2014;39(2):199-205.

26. Lin L, Hedayat AS, Wu W. Statistical Tools for Measuring Agreement, vol. 1. New York: Springer; 2012.

27. Terwee $\mathrm{CB}$, Bot $\mathrm{SD}$, de Boer MR, van der Windt DA, Knol DL, Dekker J, Bouter LM, de Vet HC. Quality criteria were proposed for measurement properties of health status questionnaires. J Clin Epidemiol. 2007;60(1):34-42.

28. Landis JR, Koch GG. An application of hierarchical kappa-type statistics in the assessment of majority agreement among multiple observers. Biometrics. 1977:33(2):363-74.

29. Carrino JA, Morrison WB, Zou KH, Steffen RT, Snearly WN, Murray PM. Lateral ulnar collateral ligament of the elbow: optimization of evaluation with twodimensional MR imaging. Radiology. 2001;218(1):118-25.

30. Carrino JA, Morrison WB, Zou KH, Steffen RT, Snearly WN, Murray PM. Noncontrast MR imaging and MR arthrography of the ulnar collateral ligament of the elbow: prospective evaluation of two-dimensional pulse sequences for detection of complete tears. Skelet Radiol. 2001;30(11):625-32.

31. Eygendaal D, Heijboer MP, Obermann WR, Rozing PM. Medial instability of the elbow: findings on valgus load radiography and MRI in 16 athletes. Acta Orthop Scand. 2000;71(5):480-3.

32. Hill Jr NB, Bucchieri JS, Shon F, Miller TT, Rosenwasser MP. Magnetic resonance imaging of injury to the medial collateral ligament of the elbow: a cadaver model. J Shoulder Elbow Surg. 2000;9(5):418-22.

33. O'Driscoll SW, Jupiter JB, King GJ, Hotchkiss RN, Morrey BF. The unstable elbow. Instr Course Lect. 2001:50:89-102.

34. Cotten A, Jacobson J, Brossmann J, Pedowitz R, Haghighi P, Trudell D, Resnick D. Collateral ligaments of the elbow: conventional MR imaging and MR arthrography with coronal oblique plane and elbow flexion. Radiology. 1997;204(3):806-12.

35. Sanchez-Sotelo J, Morrey BF, O'Driscoll SW. Ligamentous repair and reconstruction for posterolateral rotatory instability of the elbow. J Bone Joint Surg Br Vol. 2005:87(1):54-61.

36. Hackl M, Wegmann K, Ries C, Leschinger T, Burkhart KJ, Muller LP. Reliability of magnetic resonance imaging signs of posterolateral rotatory instability of the elbow. J Hand Surg. 2015;40(7):1428-33.

\section{Submit your next manuscript to BioMed Central and we will help you at every step:}

- We accept pre-submission inquiries

- Our selector tool helps you to find the most relevant journal

- We provide round the clock customer support

- Convenient online submission

- Thorough peer review

- Inclusion in PubMed and all major indexing services

- Maximum visibility for your research

Submit your manuscript at www.biomedcentral.com/submit
C) Biomed Central 